\title{
Design of a Wearable, Low-Cost, Through-Wall Doppler Radar System
}

\section{Sam Agneessens, ${ }^{1}$ Patrick Van Torre, ${ }^{1,2}$ Frederick Declercq, ${ }^{1}$ Bart Spinnewyn, ${ }^{1}$ Gert-Jan Stockman, ${ }^{1}$ Hendrik Rogier, ${ }^{1}$ and Dries Vande Ginste ${ }^{1}$}

\author{
${ }^{1}$ Department of Information Technology, Ghent University, Sint-Pietersnieuwstraat 41, 9000 Ghent, Belgium \\ ${ }^{2}$ INWE Department, Hogeschool Gent, Schoonmeersstraat 52, 9000 Ghent, Belgium
}

Correspondence should be addressed to Sam Agneessens, sam.agneessens@intec.ugent.be

Received 24 February 2012; Accepted 16 June 2012

Academic Editor: C. Aanandan

Copyright (C) 2012 Sam Agneessens et al. This is an open access article distributed under the Creative Commons Attribution License, which permits unrestricted use, distribution, and reproduction in any medium, provided the original work is properly cited.

\begin{abstract}
A novel, low-cost, low-weight, wearable Doppler radar system composed of textile materials and capable of detecting moving objects behind a barrier is presented. The system operates at $2.35 \mathrm{GHz}$ and is integrable into garments, making it well-suited for usage in difficult to access terrain, such as disaster areas or burning buildings. Wearability is maximized by relying on flexible, low-weight, and breathable materials to manufacture the key parts of the system. The low-complexity Doppler radar system makes use of an array of four textile-transmit antennas to scan the surroundings. The beam emitted by this array is right-hand circularly polarized along all scanning angles and provides a measured gain of $9.2 \mathrm{dBi}$. At the receiving end, textile materials are used to develop an active wearable receive antenna, with $15.7 \mathrm{dBi}$ gain, $1.1 \mathrm{~dB}$ noise figure, left-hand circular polarization, and a $3 \mathrm{~dB}$ axial ratio beamwidth larger than $50^{\circ}$. Several measurement setups demonstrate that the onbody system is capable of detecting multiple moving subjects in indoor environments, including through-wall scenarios.
\end{abstract}

\section{Introduction}

By integrating textile antennas into garments, low-weight, wearable, wireless systems can be created. These systems have the advantage that they are comfortable to wear and do not limit the user's ability to move. Such systems can be deployed to gather environmental (air humidity, temperature) or biometric data (heart rate, respiratory data), to provide information about the user's location (GPS), to inform the user about hazardous situations, and so forth.

An important field in which such systems can be deployed is security and rescue applications. The potential to detect and track human movers through walls and closed doors is an interesting feature for security, surveillance, and rescue systems [1]. Such an ability can be used to localize survivors after a natural disaster or accidents (e.g., an earthquake, people in a burning building) or to provide information about the position and number of criminals or hostile forces in an urban area.
For deployment during rescue operations, it is vital that the through-wall radar system is low-weight, compact, and comfortable to wear [2]. The user's motions should not be hindered and the extra effort of transporting the system should remain minimal. Therefore, the primary motivation for this paper was to design a low-weight, wearable, through-wall radar system. It makes use of textile materials to manufacture a passive transmit array, scanning the surroundings, combined with an active receive antenna. Textile materials make the system breathable, flexible, and well-suited for integration into garments.

Extensive research has been performed to develop through-wall radars and comprehensive documentation about the state-of-the-art can be found in the literature. Systems relying on Synthetic Aperture Radar (SAR) imaging focus on the emulation of a larger aperture by moving a small antenna to enhance the system resolution $[3,4]$. Others are based on multipoint sensors or arrays and exploit the different viewing angles of these antennas in order to 
compose the image [5]. These systems are often heavy, large, and energy consuming, which requires them to be vehiclemounted, making them unsuited for practical use in difficult to access terrain, such as disaster areas or burning buildings [2]. Other systems that are designed to be limited in size and weight $[6,7]$ still suffer from the problem that they require the user to carry around an extra piece of equipment. This hinders the person's movement and leads to extra gear in situations where agility is key.

Adopting textile antennas in wireless systems is gaining importance. Several case studies indicate that they are wellsuited, as mobile phone antennas, for space applications or in wireless communication links [8-10], as they offer good performance, comparable to conventional antennas, combined with interesting properties such as low-weight, low-cost, and high level of integrability into garments [11]. They can be deployed in a similar fashion as conventional antennas, allowing to construct arrays $[12,13]$ or active antennas [14].

In this paper a novel low-weight, highly integrable, wearable radar system capable of detecting moving objects behind a barrier, such as a closed door, is designed, manufactured, and thoroughly tested. Thereto several important design choices have been made, specifically the following.

(i) The scanning of the environment is performed by a right-hand circularly polarized transmit array manufactured using materials commonly found in firefighter garments.

(ii) An active textile antenna is deployed at the receiver end of the system. This active antenna is left-hand circularly polarized to minimize mutual coupling between transmitter and receiver and to reduce the negative influence of multipath. Furthermore, the integrated low-noise amplifier (LNA) increases the system's overall sensitivity.

(iii) Beam scanning is implemented by means of power dividers, electronic phase shifters, and a microcontroller. The circuit composed by these elements may be implemented on a flexible polyimide substrate, directly integrated behind the transmit array or active antenna.

The paper is organized as follows. First, the global radar system is discussed in Section 2. The principle of operation and design choices are explained, as well as the layout of the beamforming circuit. Special attention is devoted to (i) the possibilities of integrating this circuit behind the array in order to further increase the system's wearability and (ii) the time-multiplexed measurement methodology. Section 3 is dedicated to the design criteria and development of the textile antennas: a right-hand circularly polarized transmit array and an active receive antenna, based on [14], with the additional feature of being left-handed circularly polarized. In Section 4, the simulation and measurement results are presented. The performance of the textile antennas is validated and the radar system is thoroughly tested. The system is shown to be capable of detecting multiple targets behind a closed door in a free-space and an off-body setup.

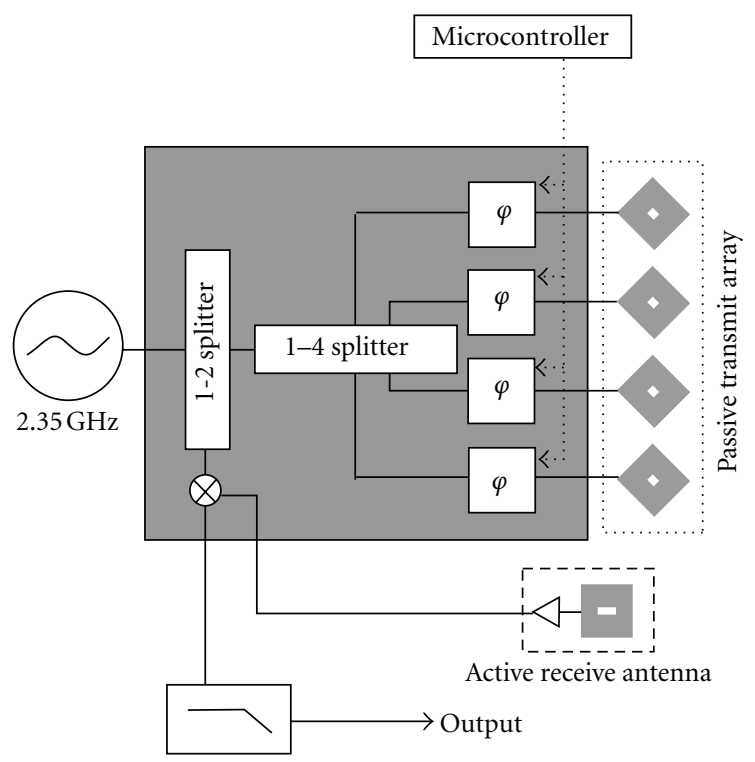

Figure 1: Block diagram of the wearable radar system.

\section{Radar System}

Textile antennas have a large number of applications. Here, they are used as the key component in the design of a low-cost, wearable, through-wall Doppler radar system. The system leverages passive and active textile antennas, that are highly integrable, making them very well-suited for a wearable radar system. To limit the system's power consumption and overall complexity, a simple Doppler architecture is deployed and data processing is kept to a minimum. Figure 1 shows the block diagram of the designed through-wall radar. The transmit side relies on a phased array of four RHCP (right-hand circularly polarized) passive antennas to electronically scan the surroundings. Steering of the beam is implemented by a beamforming circuit composed of phase shifters (JSPHS-2484+), a power divider (BP4U+), and a microcontroller, all from Mini-Circuits [15]. At the receiving end, an LHCP (left-hand circularly polarized) active antenna is used to detect the reflected signal. This signal is demodulated by a mixer (ADE-35+ from Mini-Circuits) and low-pass (LP) filter in order to extract the Doppler shift, which is then processed by a portable computer. The chosen polarizations eliminate the direct path between the transmit and receive antennas, reject secondorder reflections, and minimize the influence of multipath. The system transmits a continuous wave signal at a frequency of $2.35 \mathrm{GHz}$, avoiding interference from the $2.45 \mathrm{GHz}$ ISM band, providing good wall penetration $[16,17]$ and keeping losses in the textile materials acceptable.

The beamforming circuit is composed of the above described, small, low-weight SMT-components and, in this proof-of-principle design, it is realized on a rigid FR4 substrate. The choice for an FR4 substrate allows for easy measurement and testing during the development phase. Obviously, overall flexibility and wearability of the system 
can easily be further improved by implementing the smallfootprint circuits together with the passive interconnects on a flexible substrate. Polyimide is very well-suited for this goal, since it is a thin, low-weight, flexible substrate material that can be glued on top of a fabric layer, placed behind the ground plane of the transmit array or receive antenna. Integration of the beamforming circuit underneath the ground plane keeps the overall surface of the system small, limits the length of the interconnects - and related lossesand reduces parasitic coupling by shielding the circuit from electromagnetic radiation. This method of realizing an electronic circuit onto a hybrid polyimide-textile substrate underneath an antenna is adopted later in this paper (Section 3.2) to manufacture the active receive antenna.

Detection of moving targets is performed by adopting a time multiplexing approach. The surroundings are scanned by the transmit array at a discrete number of directions. This scanning occurs at high speed, that is, the period of a single sweep is small compared to the period of the received Doppler signal. During each sweep, the Doppler signal is sampled, each sample corresponding to a discrete transmit angle. By combining the samples of the subsequent scans for each of the directions, waveforms are formed. Each waveform corresponds to the received signal from a single direction and it is as if all the different directions are scanned "simultaneously." The sweep frequency and number of discrete sampling angles limit the maximal detectable Doppler shift. For detection of walking humans, this upper frequency does not cause problems, since the Doppler shift remains sufficiently low.

\section{Antenna Design}

A wearable radar system requires antennas that are constructed using light-weight, breathable, and flexible materials. The materials proposed below are commonly found in firefighter garments, which makes them very well-suited for the intended application. Both receive and transmit antennas are based on a rectangular ring topology with a coaxial feed [18]. This results in antennas that are easy to construct and allows obtaining circular polarization by means of a single feedpoint. Circularly polarized antennas have a number of very useful properties for the design of a radar system, such as increased isolation between receiver and transmitter and easy rejection of unwanted reflections, which makes the system less sensitive to the negative influence of multipath.

3.1. Transmit Array. The textile antenna array consists of identical rectangular ring patch antennas. The conducting patch and ground plane of the antenna elements are fabricated by means of Flectron, a copper-plated nylon fabric, with a surface resistivity of $R_{s}=0.176 \Omega /$ sq at $2.35 \mathrm{GHz}$. A polyurethane protective foam manufactured by Brunet Lion for use in firefighter suits, called Azzurri, is applied as substrate material. It has a thickness of $3.55 \mathrm{~mm}$, permittivity $\epsilon_{r}=1.19$, and loss tangent $\tan \delta=0.003$. These antennas need to be circularly polarized and matched to the $50 \Omega$ signal source, so it is required that their individual axial ratio and reflection coefficient remain below $3 \mathrm{~dB}$ and $-10 \mathrm{~dB}$, respectively.

The array itself is a phased array, this allows for simple and power-efficient beamforming. This transmit array must provide sufficient gain and an almost constant beam width for the different scanning angles. In order to fix the number of array elements, a tradeoff was made between high directivity and low side lobes on one hand and available space and low mutual coupling between elements on the other hand. An isolation between the elements of $20 \mathrm{~dB}$ is enforced. The last design criterion for the array is a constant RHCP polarization while scanning. This requires that the emitted radiation remains $\mathrm{RHCP}$ in the direction of maximal gain of the steered beam.

Based on these criteria, a four-element uniform linear array was designed, whose geometry and dimensions are presented in Figure 2 and Table 1. Spacing between the elements is $8.5 \mathrm{~cm}$, providing an array with an aperture of $33 \mathrm{~cm}$ allowing it to be fitted onto a human wearer's chest Figure 11.

3.2. Active Receive Antenna. The active receive antenna consists of a rectangular ring patch connected directly, without matching networks, to an LNA underneath its ground plane. This design approach avoids losses in components pertaining to the matching network and keeps interconnect losses low, as these degrade overall system noise performance and, hence, radar sensitivity. The radar system requires the LNA to have good noise performance, while maintaining sufficient gain. The active receive antenna as a whole should be LHCP to increase isolation between transmitter and receiver and to minimize the negative influence of second-order reflections and multipath on the system's performance. To achieve this, following design strategy is adopted: first, a suitable LNA design is selected, providing good noise performance and sufficient amplifier gain. In the next step, the antenna parameters are adapted to realize LHCP and the impedance required by the LNA for optimal noise performance.

For the LNA an ATF-54143 + e-PHEMT from Avago technologies is applied as the active element in a grounded source topology [19]. The LNA substrate is multilayered. The copper footprint is etched onto a $25 \mu \mathrm{m}$ polyimide film, that is, glued on top of an Aramid layer $\left(\epsilon_{r}=1.686, \tan \delta=\right.$ $0.015)$ of $400 \mu \mathrm{m}$. This design provides a stable LNA with sufficient gain and linearity as well as a low noise figure.

Similar to the passive transmit array, the antenna substrate is polyurethane foam and the ground plane and patch are again manufactured relying on Flectron. Antenna and LNA share this common ground plane through which both are connected by a feed line. The geometry of this active antenna is displayed in Figure 3 and Table 1 presents the antenna dimensions.

The design criteria for this receive antenna need to be met in a band around the frequency of the transmitted wave. The width of this band depends on the Doppler shift produced by a moving object (or person in this case). If the maximal velocity is assumed to be $6 \mathrm{~m} / \mathrm{s}$ [20], this Doppler shift remains well below $1 \mathrm{kHz}$ and the design criteria-good noise performance, ample gain and LHCP-only need to be 


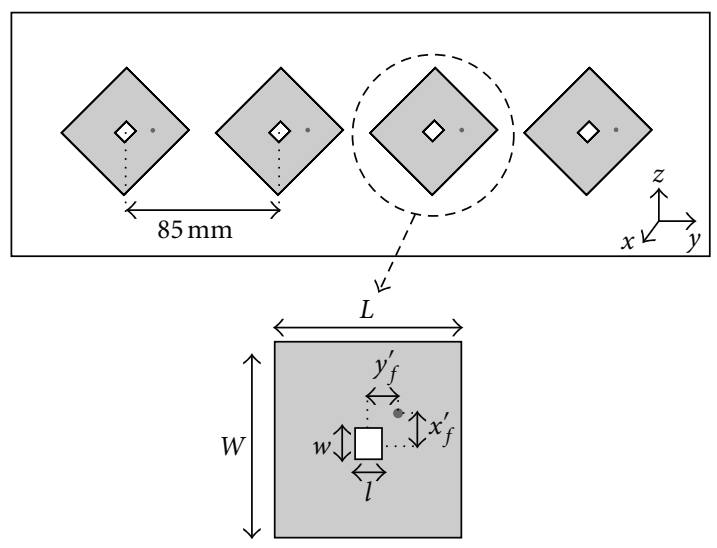

FIGURE 2: Geometry of the passive transmit array.

met in a very small band around the central frequency of $2.35 \mathrm{GHz}$.

\section{Simulations and Measurements}

4.1. Transmit Array. First, the transmit array is characterized in terms of its scattering parameters. Figure 4 shows the simulated and measured reflection coefficient for an antenna element at the edge of the array and the isolation to its direct neighbor. At $2.35 \mathrm{GHz}$, the reflection coefficient $\left|S_{11}\right|$ of the displayed antenna is smaller than $-10 \mathrm{~dB}$, indicating that the antenna elements are matched to the $50 \Omega$ signal source. The measured mutual coupling $\left|S_{21}\right|$ between the elements is lower than $-20 \mathrm{~dB}$, confirming that the isolation between these two elements is sufficiently high. The simulated and measured results for the other array elements are similar to the results shown in Figure 4.

The array radiation pattern is another important parameter of the system, as variation in the gain pattern's main lobe, deformation of the beam and size of the sidelobes have a large influence on its performance (e.g., the maximal obtainable scanning resolution). Figure 5 presents the simulated and measured gain pattern when the main lobe of the beam is steered along $0^{\circ}$ in the azimuth plane, that is, the $X Y$-plane (Figure 2). The patterns show a large degree of resemblance, but the measured gain value of $9.2 \mathrm{dBi}$ is smaller than the simulated gain of $13.8 \mathrm{dBi}$. This is due to the fact that the simulation does not take the losses in the steering circuit and the insertion loss of the phase shifters, which were measured to add up to $3.9 \mathrm{dBi}$ for a $0^{\circ}$ steering angle, into account. The measured $3 \mathrm{~dB}$ half beamwidth is $9^{\circ}$ and remains constant along all considered steering angles.

The phase shifters limit the maximal angle along which the beam can be steered, without large deformation in shape. The steering angle of the beam ranges from $-15^{\circ}$ to $+15^{\circ}$ and Figure 6 shows the measured gain pattern for the steering angles $-10^{\circ}, 0^{\circ}$ and $15^{\circ}$. The highest measured sidelobe gain is about $2 \mathrm{dBi}$ when the beam is steered along an angle of $\pm 15^{\circ}$, resulting in a sidelobe suppression of $7 \mathrm{~dB}$, which is important in avoiding "ghost echoes." The detection range can be further augmented by cascading the used phase

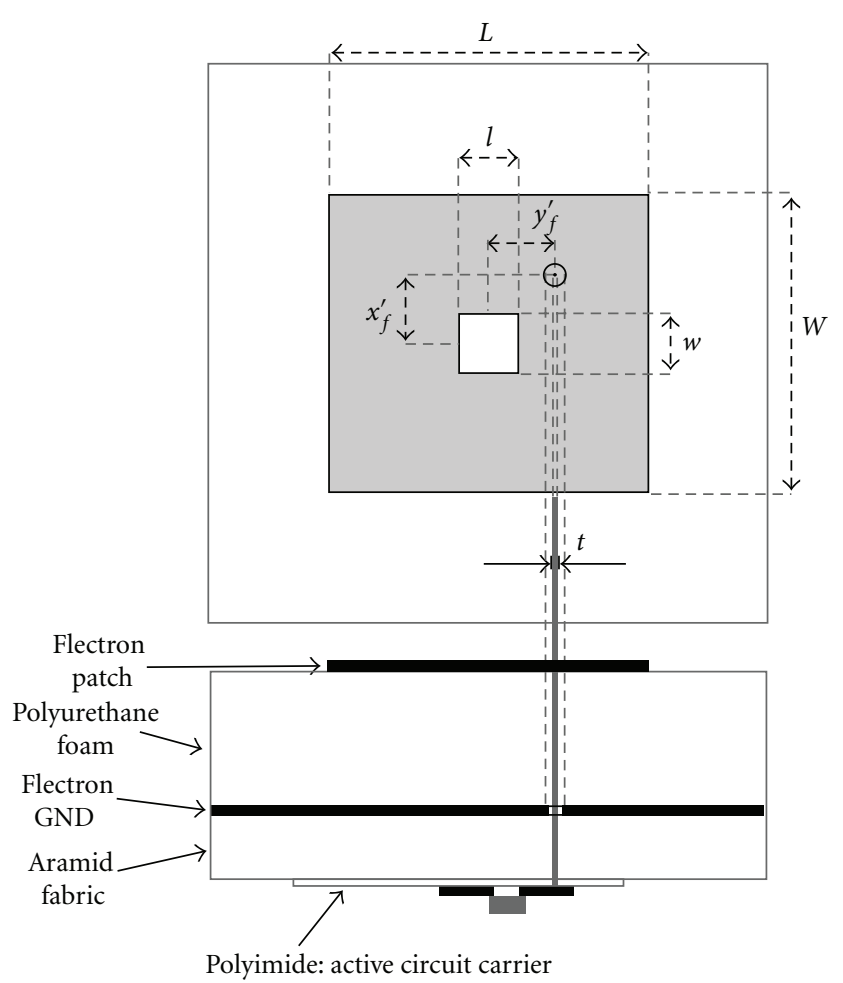

FIGURE 3: Geometry of the active receive antenna.

TABLE 1: Dimensions of the individual transmit array elements and active receive antenna element.

\begin{tabular}{lcc}
\hline & Array element & Active antenna \\
\hline$W$ & $51.8 \mathrm{~mm}$ & $49.5 \mathrm{~mm}$ \\
$L$ & $55.4 \mathrm{~mm}$ & $51 \mathrm{~mm}$ \\
$w$ & $8.8 \mathrm{~mm}$ & $17.5 \mathrm{~mm}$ \\
$l$ & $7.5 \mathrm{~mm}$ & $7 \mathrm{~mm}$ \\
$x_{f}^{\prime}$ & $7.4 \mathrm{~mm}$ & $8 \mathrm{~mm}$ \\
$y_{f}^{\prime}$ & $10.1 \mathrm{~mm}$ & $-5 \mathrm{~mm}$ \\
$t$ & $1.25 \mathrm{~mm}$ & $0.85 \mathrm{~mm}$ \\
\hline
\end{tabular}

shifters. Simulations indicate that the deformation of the beam at a maximal steering angle of $\pm 30^{\circ}$ remains low and a side lobe suppression larger than $8.5 \mathrm{~dB}$, so the scanning range may be increased to cover $-30^{\circ}$ to $+30^{\circ}$ without system performance loss.

Another important feature of the system is the polarization of the beam. Figure 7 visualizes the measured axial ratio for different steering angles. It can be seen that the array remains RHCP along all these angles.

4.2. Active Receive Antenna. The active receive antenna is designed to have an impedance matched to the simulated optimal noise impedance of the LNA, that is, (35.7- j15.7) $\Omega$, to maximize the noise performance. This antenna without LNA has a measured gain, $G_{p}$, in the main direction of $7.3 \mathrm{dBi}$. The simulated and measured gain pattern, $G_{\text {tot }}$, and axial ratio of the active receive antenna are shown in Figure 8. The maximal simulated and measured gain is $18.6 \mathrm{dBi}$ and 

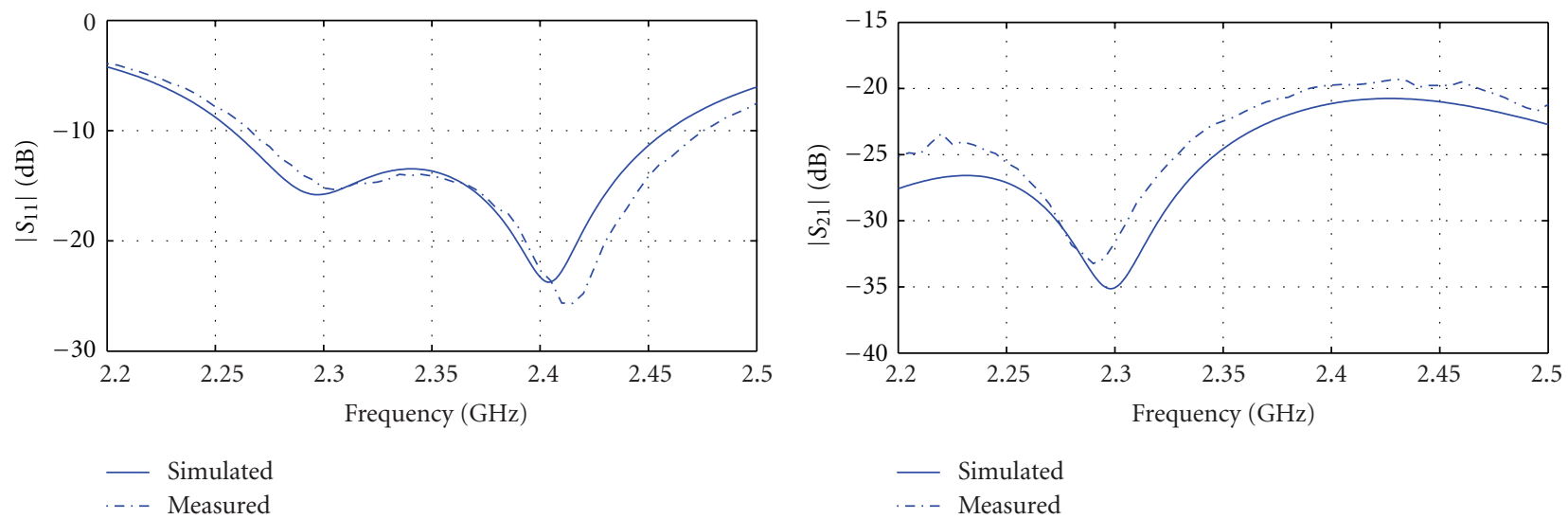

FIGURE 4: Simulated (full line) and measured (dashed-dotted line) reflection coefficient of a single element and mutual coupling between two neighboring array elements.

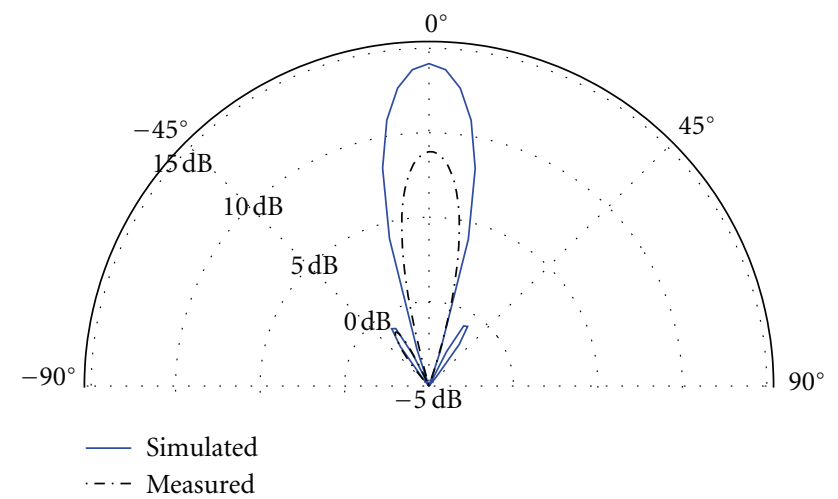

FIGURE 5: Simulated (full line) and measured (dashed-dotted line) gain pattern in the azimuth plane, that is, the $X Y$-plane (Figure 2), for an array with main lobe steered along $0^{\circ}$.

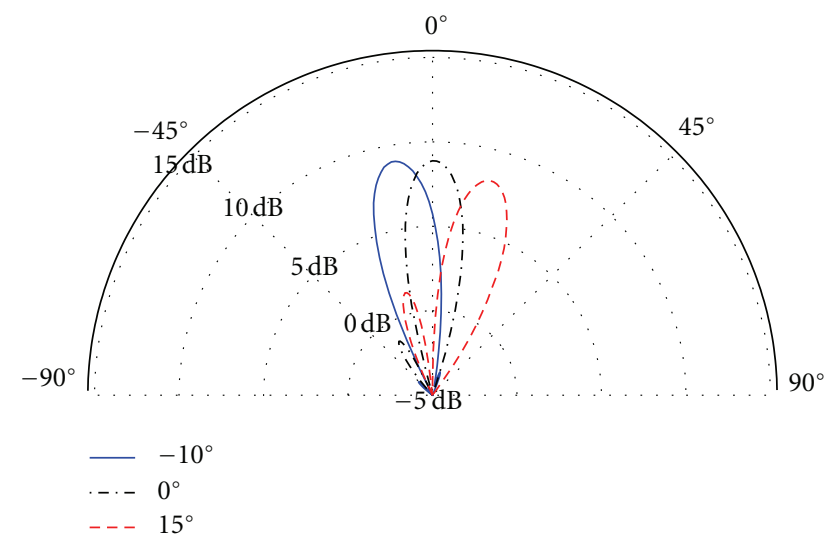

FIGURE 6: Radiation pattern in the azimuth plane with direction of maximal gain at $-10^{\circ}, 0^{\circ}$, and $15^{\circ}$.

$15.7 \mathrm{dBi}$, respectively. The measured $3 \mathrm{~dB}$ beamwidth in terms of gain exceeds $60^{\circ}$ and the $3 \mathrm{~dB}$ axial ratio beamwidth in terms of circular polarization is larger than $50^{\circ}$. The discrepancies in gain and axial ratio between measurements

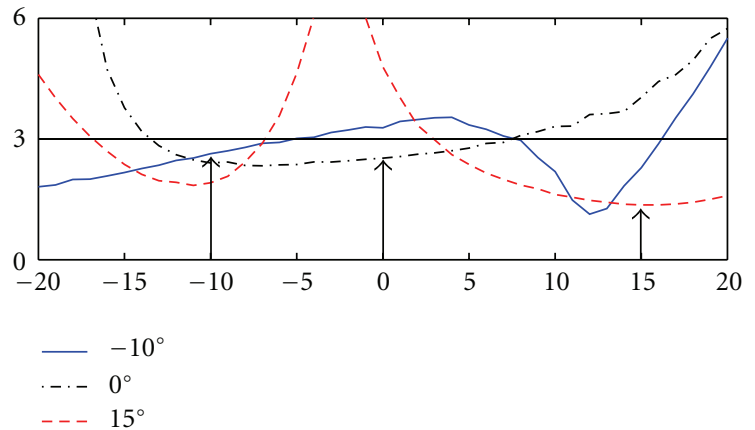

Figure 7: Axial ratio for radiation patterns in the azimuth plane with direction of maximal gain at $-10^{\circ}, 0^{\circ}$, and $15^{\circ}$.

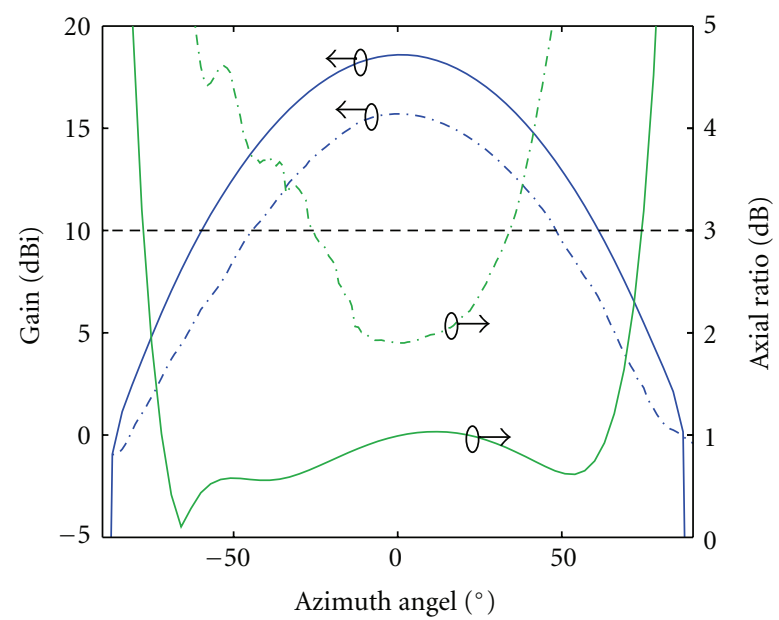

FIGURE 8: Simulated (full line) and measured (dashed-dotted line) total gain and axial ratio for the active receive antenna.

and simulations evolve from the manual manufacturing process and misalignments during the measurements.

The LNA is unconditionally stable and, when attached to the receive antenna, exhibits a simulated transducer gain 
of $11.3 \mathrm{~dB}$ and a noise figure of $0.9 \mathrm{~dB}$ at the operating frequency of $2.35 \mathrm{GHz}$ when attached to the receive antenna. The actual transducer gain, $G_{t}$ of the LNA cannot be measured directly, due to the integration into the active antenna, but it can be deducted from following relation:

$$
G_{\mathrm{tot}}(\theta, \phi)=G_{p}(\theta, \phi) G_{T},
$$

where $G_{\text {tot }}(\theta, \phi)$ is the measured gain of the active antenna and $G_{p}(\theta, \phi)$ is the measured gain of a passive antenna with identical dimensions. This leads to $G_{t}=8.1 \mathrm{~dB}$. With the knowledge of $G_{t}$ it is possible to calculate the noise factor by measuring the noise power density $P_{n}$ referred to $290 \mathrm{~K}$ [21]:

$$
F=1+\frac{P_{n}}{G_{t}}-\frac{T_{a}}{290}
$$

with $T_{a}$ the effective noise temperature $(286.3 \mathrm{~K})$. The noise figure measured in an anechoic chamber at $2.35 \mathrm{GHz}$ is $1.1 \mathrm{~dB}$, which is in good agreement with the simulations.

In summary, the performance of the active antenna is outstanding, making it ideal for usage as a receive antenna in the radar system.

4.3. Radar System. To validate the radar's performance, a series of measurements are conducted. First, they are carried out with the radar in a free-space environment, that is, without the presence of a human body. Next, the system is worn by a person. For both configurations, two series of measurements are performed. First, loudspeakers producing an audio tone at a fixed frequency are detected, as this yields stable and repeatable results. Next, human subjects are used as moving targets.

4.3.1. Free-Space Measurements. The transmitter and receiver are placed in free-space at a distance of approximately $20 \mathrm{~cm}$ apart from each other. As such, the measured isolation between them is larger than $40 \mathrm{~dB}$, which is sufficient for the radar system to function properly. This low coupling avoids saturation of the active antenna amplifier by the power of the transmitted wave, which is $5 \mathrm{dBm}$.

The first experiment uses a single speaker, producing a fixed tone at a low frequency $(10 \mathrm{~Hz})$, so that the movement of the cone is large enough to measure. The speaker is placed along a $0^{\circ}$ azimuth angle, $3 \mathrm{~m}$ away from the radar and the cone is covered by copper tape to increase the reflections. The received spectrum for each angle is visualized in Figure 9. The $10 \mathrm{~Hz}$ peak is clearly visible at $0^{\circ}$ and only noise is received along the other scanned angles.

Figure 10 shows the frequency spectrum for the second experiment. There are two different targets: a person approaches the radar at the $0^{\circ}$ angle and a speaker is placed at the $-15^{\circ}$ angle, now producing a $15 \mathrm{~Hz}$ tone. The human target is detected at a peak frequency of $24 \mathrm{~Hz}$, which corresponds to a normal walking velocity of $1.5 \mathrm{~m} / \mathrm{s}$. The speaker is picked up at $-15^{\circ}$. There is also a smaller "ghost echo," originating from the sidelobes of the scanning beam at $15^{\circ}$.

4.3.2. On-Body Measurements. The influence of the wearer on the system's ability to detect targets behind a closed
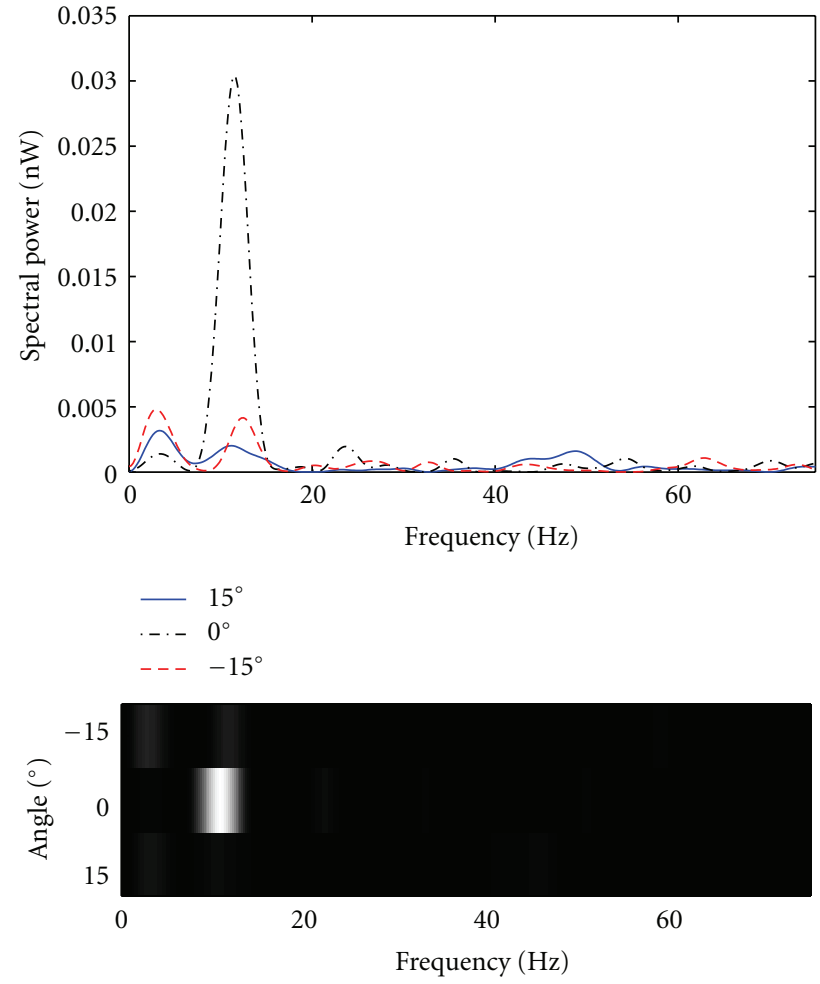

FIGURE 9: Spectrogram of the received signal when a speaker, producing a $10 \mathrm{~Hz}$ tone, is placed at $0^{\circ}$.
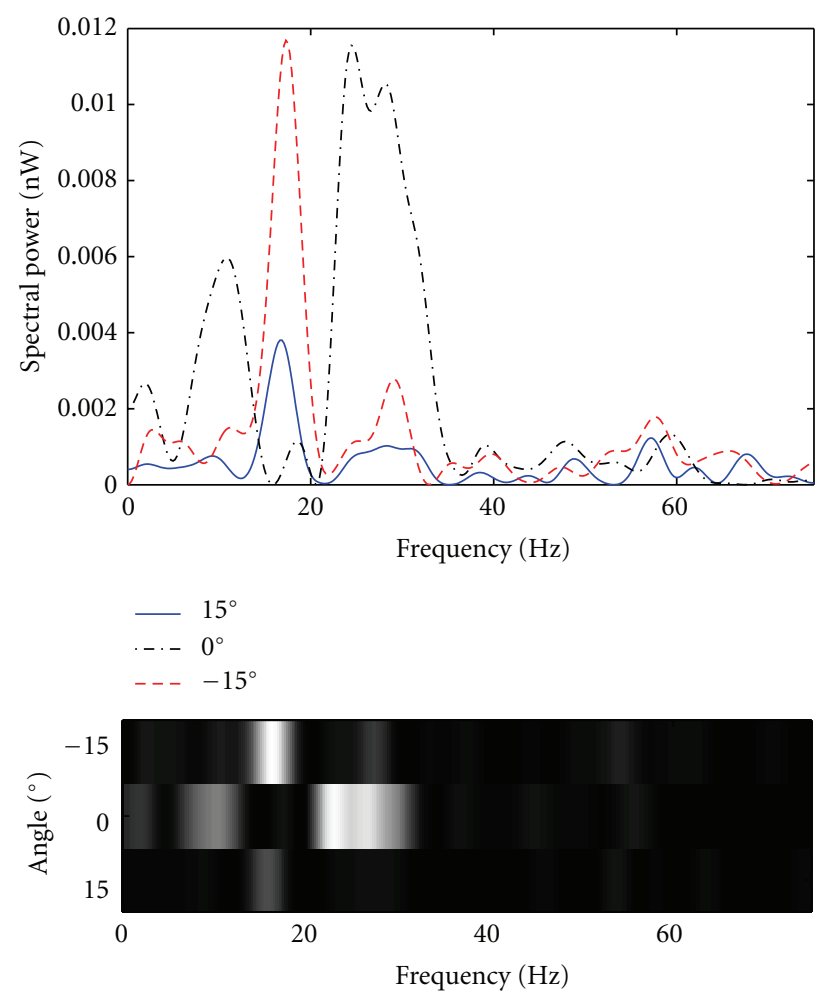

FIGURE 10: Spectrogram for a person walking towards the radar at a $0^{\circ}$ angle and a speaker placed at $-15^{\circ}(15 \mathrm{~Hz})$. 


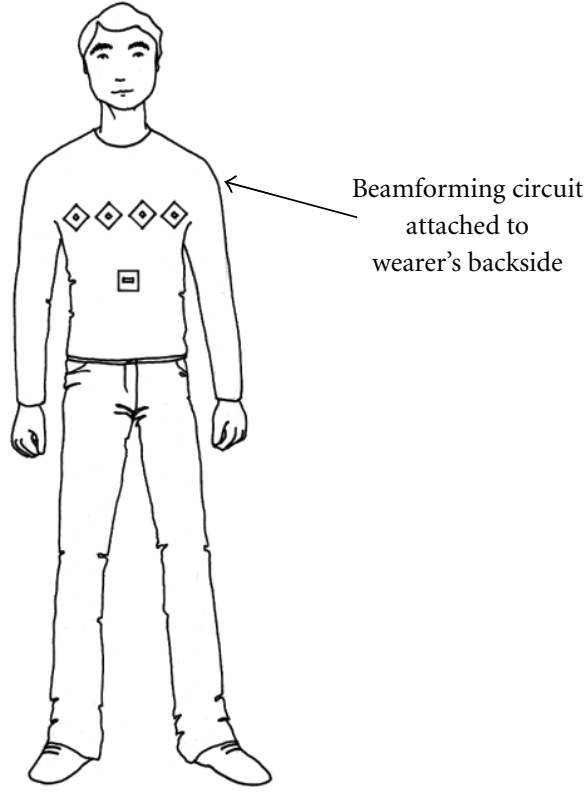

FIgure 11: Textile radar system positioned on a human wearer (drawing by Laura Goethals).

wooden door, covered by fire retardant paint, is analyzed. Figure 11 gives a representation of how the system is positioned on a human wearer. The array is attached onto the user's chest in order to minimize the deformation of the array and the active antenna is placed below it at a large enough distance in order to limit mutual coupling between transmitter and receiver. The beamforming circuit is attached to the back of the suit.

The person wearing the radar is separated from the target by a barrier, in our experiment a closed wooden door. The targets used in the experiments consist of a speaker producing a $15 \mathrm{~Hz}$ tone placed at approximately $1.5 \mathrm{~m}$ and a person walking towards the radar at the other side of the door. The frequency spectra measured during these tests are shown in Figure 12 (speaker) and Figure 13 (person), respectively. The results are similar to the free-space experiments, the frequency components related to the movement of the speaker and human target are clearly visible. This indicates that the system performance is not significantly reduced when deployed onto a human wearer and that detection remains possible even in the presence of a barrier, such as a door or a wall, between the radar and the moving object.

Deploying the radar on the human body as described in this paper limits the region where objects can be detected to the frontal hemisphere. However, certain cases may require the wearer to have knowledge of what is happening behind him/her. The time-multiplexed scanning method allows simple extension of the detection area, to include the backward hemisphere, by the addition of an extra transmit array and receiver antenna at the rear of the observer, but without the need of extra data processing hardware. This extended detection range comes at the cost of additional scanning angles and may require an increase of scanning and sampling frequency to maintain a usable (i.e., high enough)
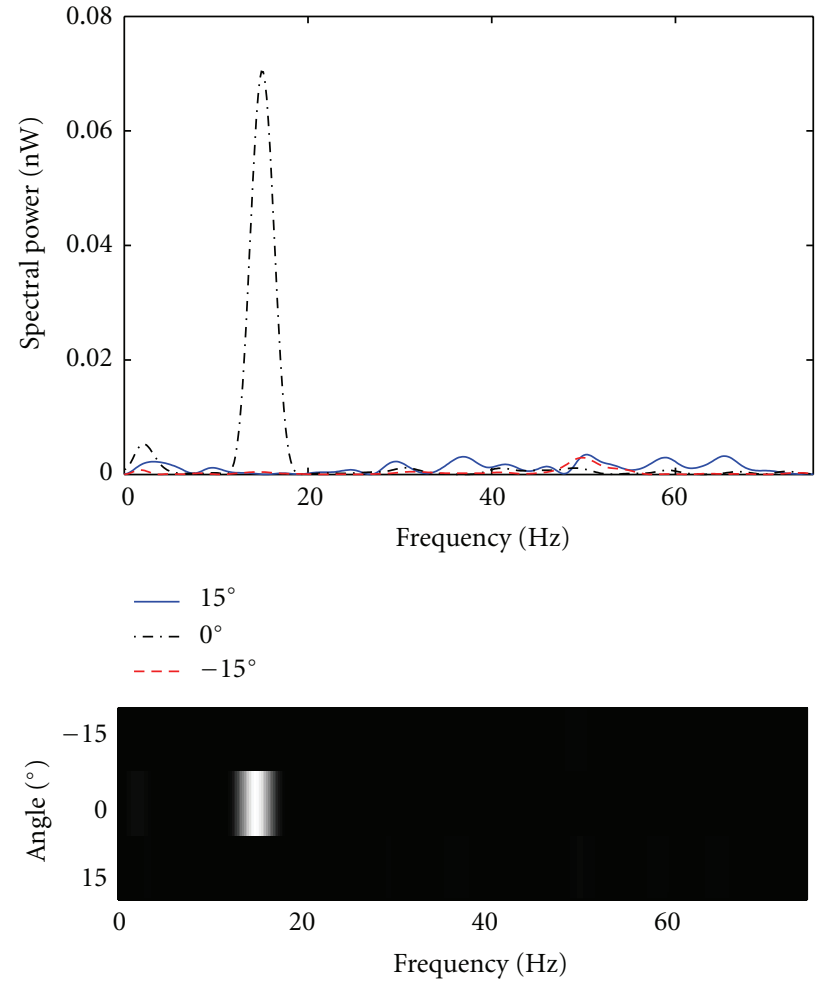

FIGURE 12: Spectrogram for a speaker at distance of $1.5 \mathrm{~m}$, producing a $15 \mathrm{~Hz}$ tone behind a closed door when the radar is deployed on the human body.
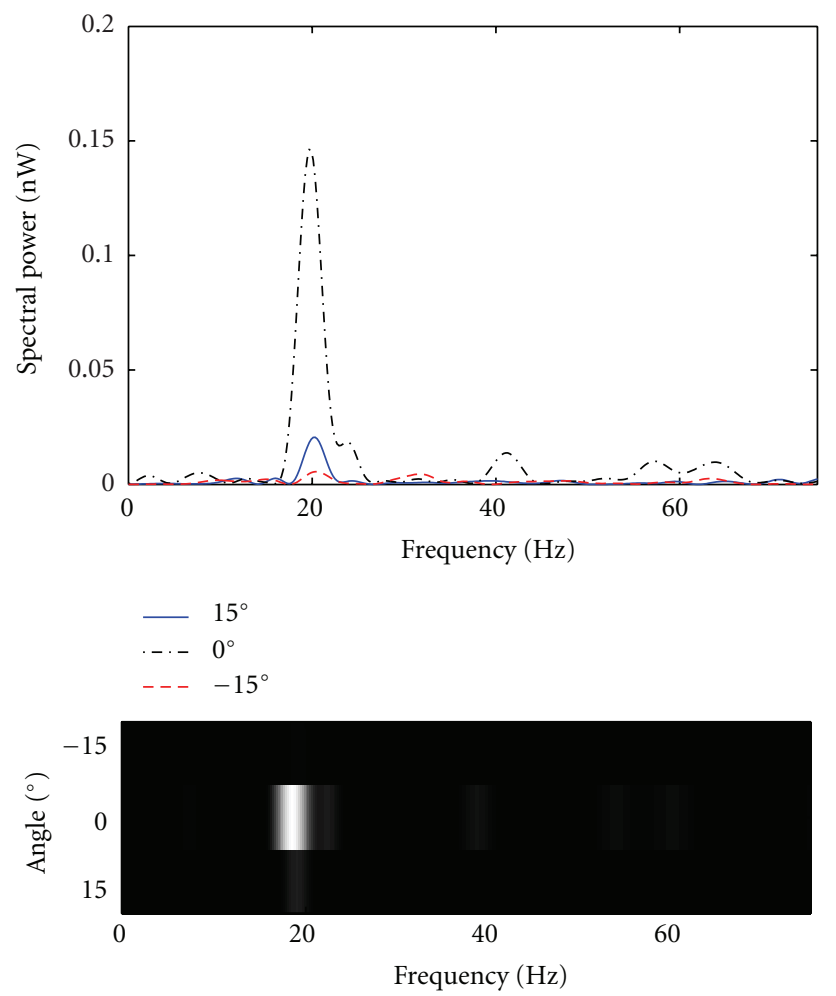

FIGURE 13: Spectrogram for a person walking towards the observer behind a closed door when the radar is deployed on the human body. 
maximal detectable Doppler shift. A system which relies on such a front and back array to increase its performance is presented in [22].

\section{Conclusions and Future Research}

A novel low-weight, wearable radar system, capable of detecting moving human subjects behind a barrier, such as a door, a wall, or debris of a building, was proposed.

Wearability is maximized by using textile materials to manufacture the receive and transmit antennas. The RHCP transmit array allows to scan the environment and maintain a circular polarization along all steering angles. The system sensitivity is enhanced by using an LHCP active receive antenna, optimized for noise performance. Using opposite antenna polarizations enhances the isolation between receiver and transmitter, allows for easy rejection of second-order reflections and minimizes the unwanted effects of multipath. Furthermore, the beamforming circuit is small and can easily be realized on a flexible substrate, such as polyimide, which can be glued behind the array to improve wearability and to limit the system's size. The extensive use of breathable, low-weight and flexible materials in the design of this radar system makes it very well-suited to be integrated into garments worn by rescue workers, firefighters, or law enforcement personnel operating in rough, difficult to access, or hazardous terrain.

The main individual components of the radar system, that is, the transmit array and active receive antenna, as well as the system itself, have been thoroughly tested, validating the design. The main novelty and focus of this work is, however, the wearability of the system and it was a deliberate design choice to keep the radar data processing simple and power-efficient.

Better performance can easily be obtained by more extensive data processing or the work can be taken as a starting point for a wearable radar system with imaging techniques. Further study including measurements in scenarios where the targets are behind multilayered structures, such as concrete or composite walls, is needed to further characterize the system's performance as a through-wall radar system. Such future work can also include the study of the system's performance when it is worn in more dynamic situations which may cause more bending or deformations of the transmit array and receiver antenna (e.g., crouching).

\section{References}

[1] E. J. Baranoski, "Through-wall imaging: historical perspective and future directions," Journal of the Franklin Institute, vol. 345, no. 6, pp. 556-569, 2008.

[2] M. Farwell, J. Ross, R. Luttrell, D. Cohen, W. Chin, and T. Dogaru, "Sense through the wall system development and design considerations," Journal of the Franklin Institute, vol. 345, no. 6, pp. 570-591, 2008.

[3] F. Ahmad and M. G. Amin, "Multi-location wideband synthetic aperture imaging for urban sensing applications," Journal of the Franklin Institute, vol. 345, no. 6, pp. 618-639, 2008.
[4] M. Dehmollaian and K. Sarabandi, "Refocusing through building walls using synthetic aperture radar," IEEE Transactions on Geoscience and Remote Sensing, vol. 46, no. 6, pp. 1589-1599, 2008.

[5] F. Ahmad, M. G. Amin, and G. Mandapati, "Autofocusing of through-the-wall radar imagery under unknown wall characteristics," IEEE Transactions on Image Processing, vol. 16, no. 7, pp. 1785-1795, 2007.

[6] K. Browne, R. Burkholder, and J. Volakis, “Through-wall radar imaging system utilizing a light-weight low-profile printed array," in Proceedings of the 4th European Conference on Antennas and Propagation (EuCAP '10), pp. 12-16, IEEE, Barcelona, Spain, April 2010.

[7] S. S. Ram and H. Ling, "Through-wall tracking of human movers using joint doppler and array processing," IEEE Geoscience and Remote Sensing Letters, vol. 5, no. 3, pp. 537$541,2008$.

[8] P. J. Massey, "Mobile phone fabric antennas integrated within clothing," in Proceedings of the 11th International Conference on Antennas and Propagation, no. 480, pp. 17-20, Manchester, UK, April 2001.

[9] T. Kennedy, P. Fink, A. Chu, and G. Studor, "Potential space applications for body-centric wireless and E-textile antennas," in Proceedings of the Institution of Engineering and Technology Seminar on Antennas and Propagation for BodyCentric Wireless Communications, pp. 77-83, The Institution of Engineering and Technology, London, UK, April 2007.

[10] M. Klemm and G. Troester, "Textile UWB antennas for wireless body area networks," IEEE Transactions on Antennas and Propagation, vol. 54, no. 11, pp. 3192-3197, 2006.

[11] C. Hertleer, H. Rogier, L. Vallozzi, and L. van Langenhove, "A textile antenna for off-body communication integrated into protective clothing for firefighters," IEEE Transactions on Antennas and Propagation, vol. 57, no. 4, pp. 919-925, 2009.

[12] T. F. Kennedy, P. W. Fink, A. W. Chu et al., "Body-worn Etextile antennas: the good, the low-mass, and the conformal," IEEE Transactions on Antennas and Propagation, vol. 57, no. 4, pp. 910-918, 2009.

[13] M. L. Scarpello, D. Vande Ginste, and H. Rogier, "Design of a low-cost steerable textile antenna array operating in varying relative humidity conditions," Microwave and Optical Technology Letters, vol. 54, no. 1, pp. 40-44, 2012.

[14] F. Declercq and H. Rogier, "Active integrated wearable textile antenna with optimized noise characteristics," IEEE Transactions on Antennas and Propagation, vol. 58, no. 9, pp. 30503054, 2010.

[15] http://www.minicircuits.com/.

[16] M. Amin and K. Sarabandi, "Special issue on remote sensing of building interior," IEEE Transactions on Geoscience and Remote Sensing, vol. 47, no. 5, pp. 1267-1268, 2009.

[17] T. B. Gibson and D. C. Jenn, "Prediction and measurement of wall insertion loss," IEEE Transactions on Antennas and Propagation, vol. 47, no. 1, pp. 55-57, 1999.

[18] G. Vermeeren, H. Rogier, F. Olyslager, and D. de Zutter, "Low-cost planar rectangular ring antenna for operation in HiperLAN band," Electronics Letters, vol. 38, no. 5, pp. 208209, 2002.

[19] ATF-54143, "High Intercept Low Noise Amplifier for the 1850-1910 MHz PCS Band using the Enhancement Mode PHEMT," Application Note 1222, Avago Technologies, 2006.

[20] S. S. Ram, Y. Li, A. Lin, and H. Ling, "Doppler-based detection and tracking of humans in indoor environments," Journal of the Franklin Institute, vol. 345, no. 6, pp. 679-699, 2008. 
[21] H. An, B. Nauwelaers, A. R. van de Capelle, and R. G. Bosisio, "A novel measurement technique for amplifier-type active antennas," in Proceedings of the IEEE MTT-S International Microwave Symposium Digest, vol. 3, pp. 1473-1476, San Diego, Calif, USA, May 1994.

[22] P. van Torre, L. Vallozzi, H. Rogier, and J. Verhaevert, "Indoor off-body wireless communication using static zero-elevation beamforming on front and back textile antenna arrays," in Proceedings of the 6th European Conference on Antennas and Propagation (EuCAP '12), Prague, Czech Republic, March 2012, Convened Session "Recent Advances and Applications of Body-Centric Wireless Communications". 

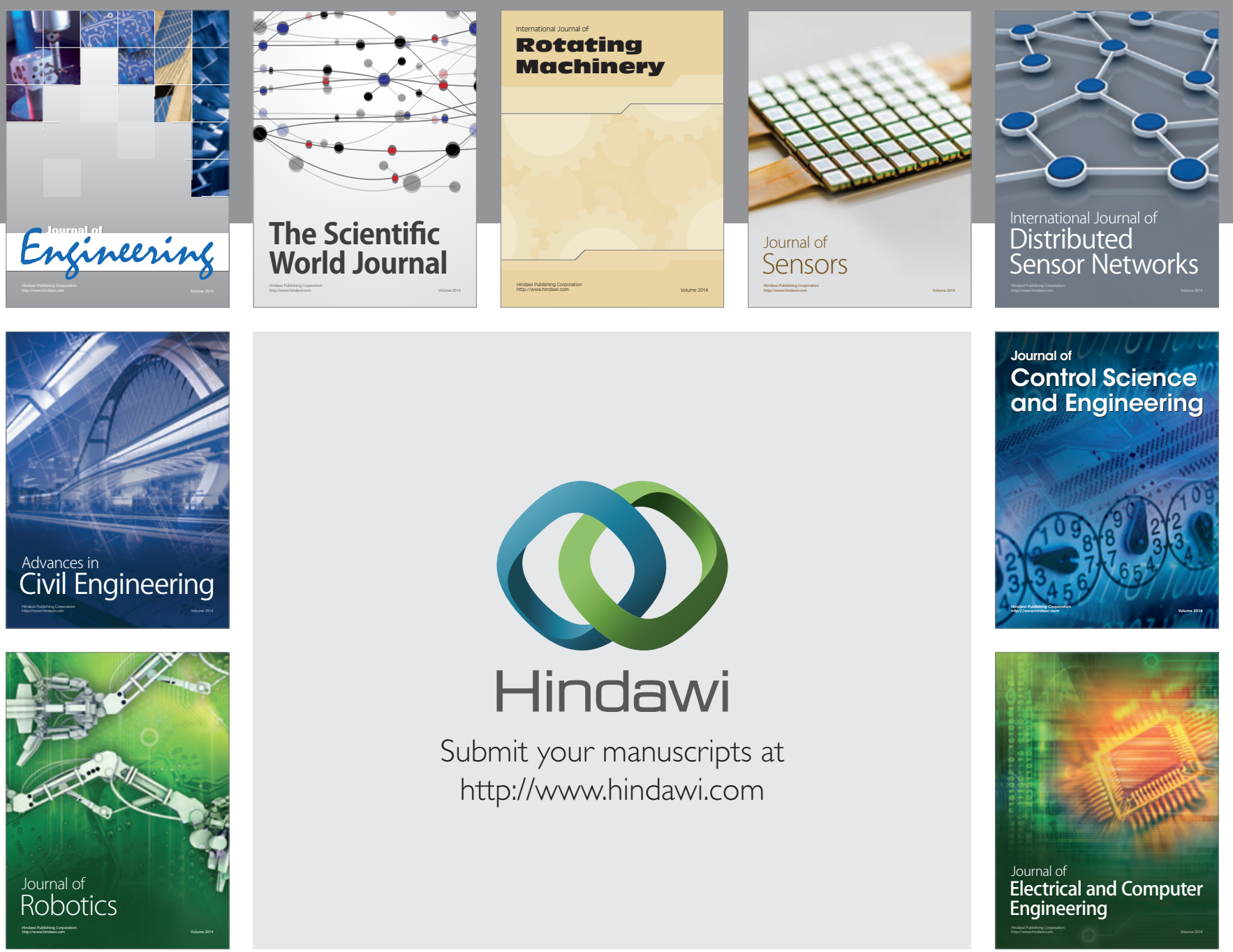

Submit your manuscripts at

http://www.hindawi.com
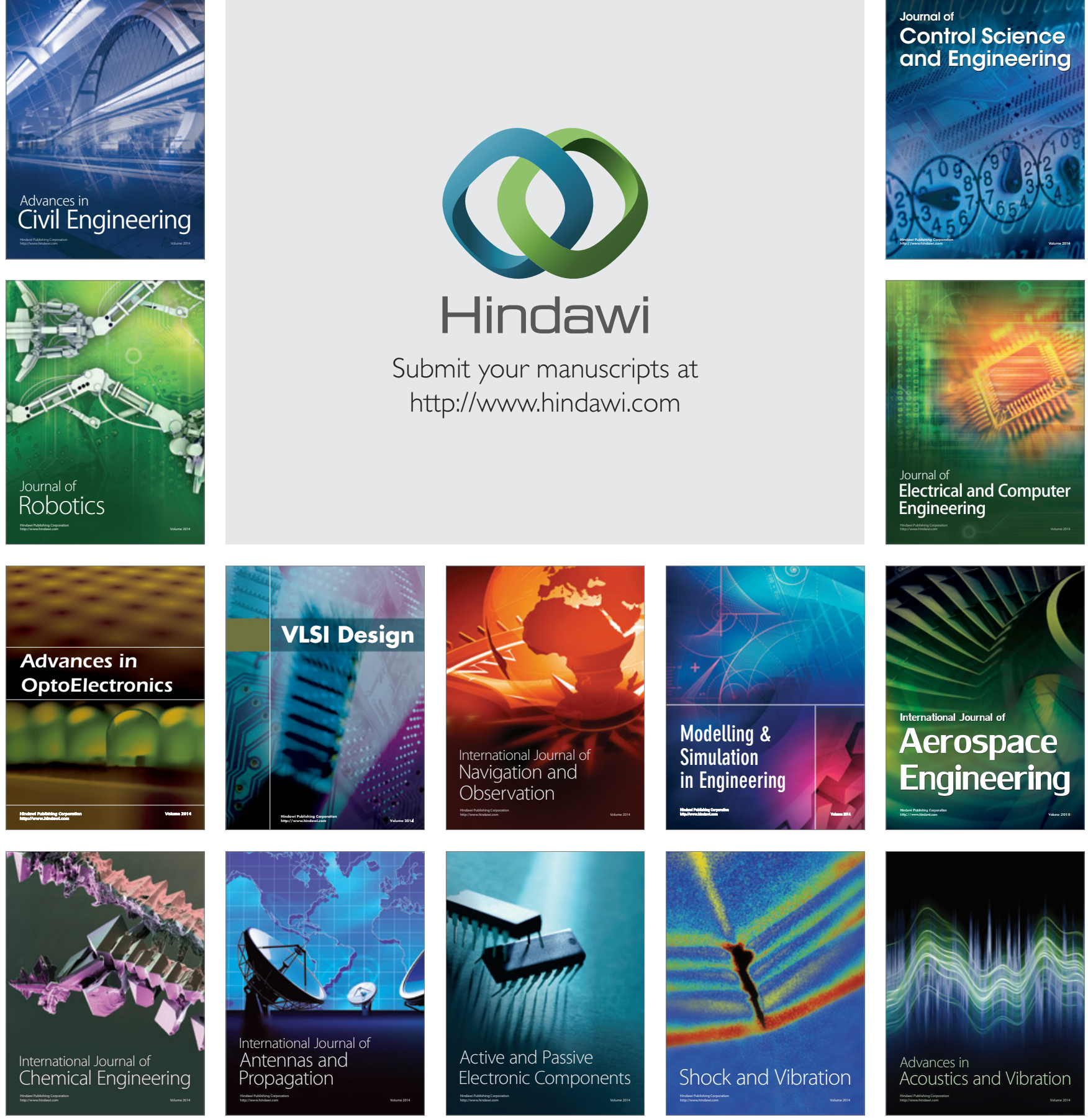